\title{
Low-Grade Follicular Lymphoma with Highly Unusual Flow Cytometric, FISH, Molecular, and Classical Cytogenetic Results: A Case Report
}

\author{
Jian Jeff Fu ${ }^{1 *}$, Yulan Gong ${ }^{1}$ and Joseph M Jaworski ${ }^{2 *}$ \\ ${ }^{1}$ Department of Pathology, Fox Chase Cancer Center, USA \\ ${ }^{2}$ Department of Pathology, Mercy Fitzgerald Hospital, USA \\ *Corresponding author: Jian Jeff Fu, Department of Pathology, Fox Chase Cancer Center, hiladelphia, USA
}

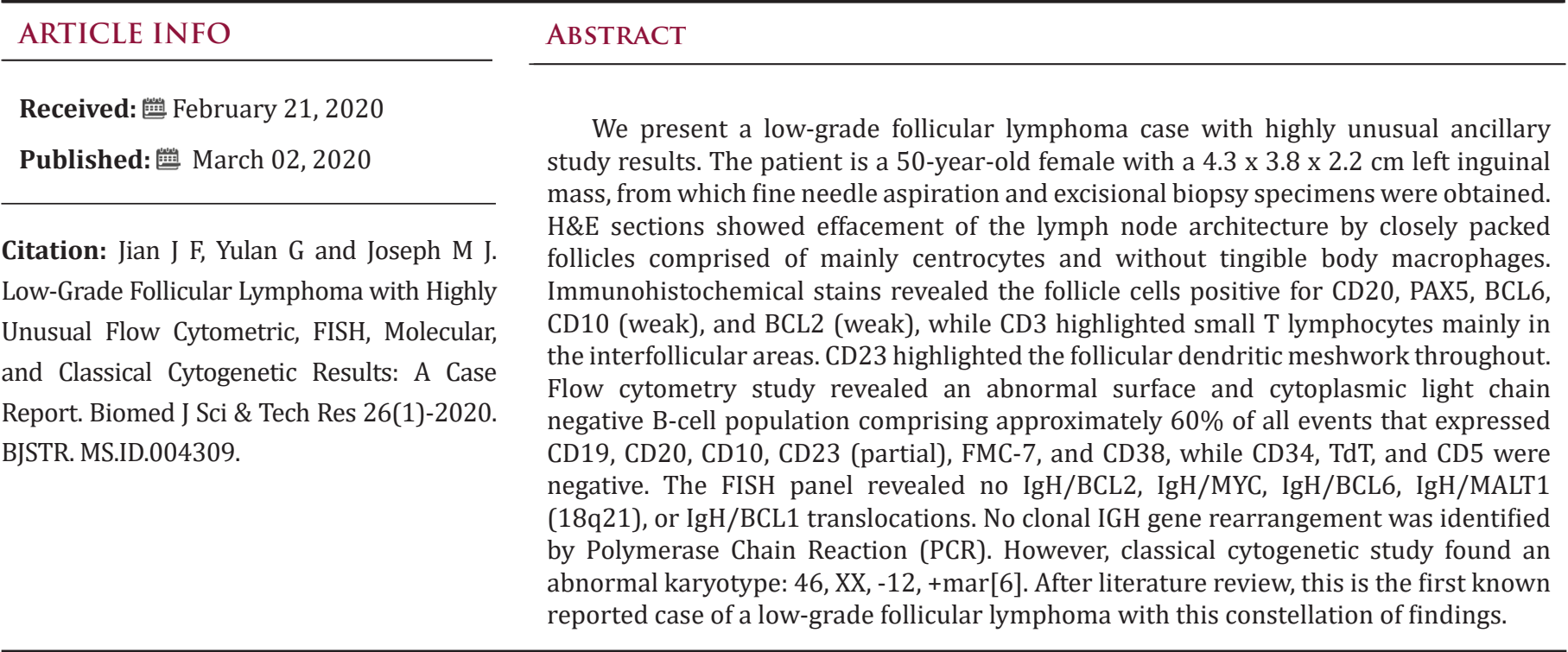

\section{Introduction}

Follicular Lymphoma (FL) is the second most common Non-Hodgkin Lymphoma (NHL) with the highest incidence in Western countries [1,2]. The diagnosis of FL is commonly based on the morphology as well as immunophenotyping with immunohistochemical staining and flow cytometry, and sometimes molecular studies. Follicular and diffuse patterns are recognized with a varying proportion of each that may be seen in a lymph node. The most predominant pattern is follicular with closely packed follicles that efface the nodal architecture. The FL cells usually express Surface Immunoglobulin (sIg), B cell antigens, BCL2, BCL6, and CD10, and are negative for CD5 and CD43. Genetically, FL is usually characterized by the translocation t $(14 ; 18)$ (q32; q21), especially in low-grade FL. Clonality detection by PCR analysis of immunoglobulin heavy and light chains may provide further support for the diagnosis. Of interest, the demonstration of sIg light chain restriction for monoclonality is not always achieved in malignant B cell NHL. Although it is rare, sIg-negative NHL has been reported and lack of sIg light chain expression may help diagnose peripheral B cell lymphoma $[3,4]$ We report a case of a lymph node involved by low-grade FL without surface or cytoplasmic light chain expression, negative Ig heavy chain clonal rearrangement by PCR, negative IgH/BCL2 translocation by FISH, and an abnormal karyotype of 46,XX,-12,+mar[6].

\section{Case report}

A 50-year-old female patient with past medical history of hypertension, hyperlipidemia, peripheral vascular disease, coronary atherosclerosis, obstructive sleep apnea, esophageal 
reflux, and lumbar disc displacement complained of a left groin mass and left leg pain that had been persistent for 3 to 4 months. CT scan of pelvis was performed, revealing an enlarged left inguinal lymph node measuring $4.3 \times 3.8 \mathrm{~cm} \times 2.2 \mathrm{~cm}$ and a few additional prominent left inguinal lymph nodes. Ultrasound-guided fine needle aspiration was obtained from the left inguinal node and subjected to flow cytometry analysis. There was an abnormal B-cell population comprising approximately $60 \%$ of the total events which expressed CD19, CD20, FMC7, CD10, CD38, and CD23 (partial), and was negative for CD5, TdT, and CD34. There was no expression of surface or cytoplasmic light chains. Therefore, these atypical lymphoid cells were suspicious for lymphoproliferative disorder and the differential diagnosis included FL, diffuse large B-cell lymphoma, and Burkitt lymphoma. The patient's complete blood count was unremarkable.One month later, excisional biopsy of the left inguinal node was performed, and parts of the specimen were sent for flow cytometry, NHL Fluorescence In Situ Hybridization (FISH) panel, IGH rearrangement by PCR, and cytogenetics in addition to routine $\mathrm{H} \& \mathrm{E}$ and immunohistochemical stains.
Flow cytometry demonstrated an identical result to that of the previous fine needle aspiration. Morphologically, numerous follicles were closely packed, lacked mantle zones, and showed a back-to-back pattern at low power (Figure 1A), consistent with follicular pattern of FL. CD23 highlighted the follicular dendritic cell meshwork throughout (Figure 1B). At high power, mainly centrocytes and few centroblasts were distributed in the follicles and tingible body macrophages were absent (Figure 1C). Immunohistochemical stains showed the follicle cells positive for CD20, BCL6, CD10 (weak), and BCL2 (weak) (Figures 1D-1K), while $\mathrm{CD} 3$ highlighted small $\mathrm{T}$ lymphocytes mainly in the interfollicular areas (Figure 1H) and sparsely in the follicular areas (Figure 1I). CD10 expression was confirmed by flow cytometry. The FISH panel revealed no IgH/BCL2, IgH/MYC, IgH/BCL6, IgH/BCL1, and IgH/ MALT1 (18q21) translocations. No clonal IGH gene rearrangement was identified by PCR. However, cytogenetic study found a clonal abnormality in all 6 analyzable cells, demonstrating an abnormal karyotype of $46, \mathrm{XX},-12,+\operatorname{mar}[6]$.

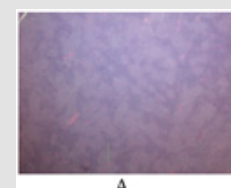

A.

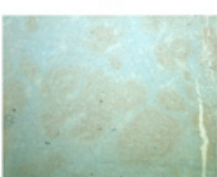

E.

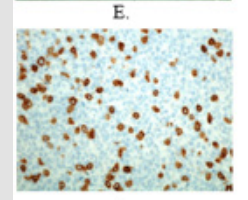

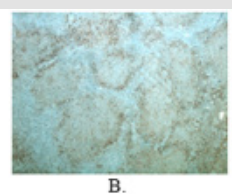

B
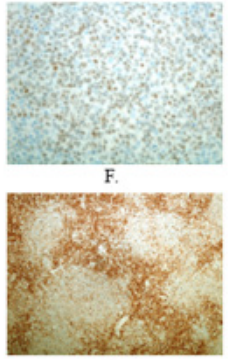
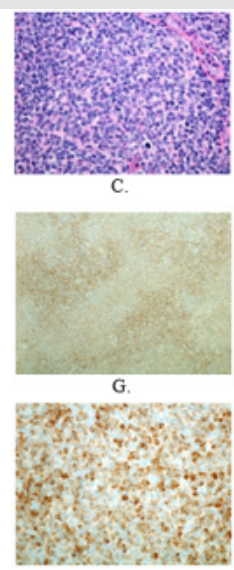
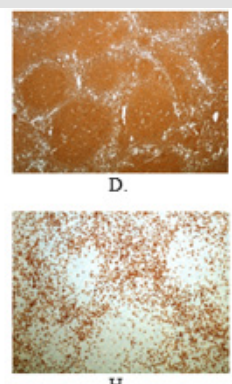

H.

Figure 1: A, C: H\&E showing effacement of lymph node architecture by closely packed follicles (A: 2x; C: 40x). B, D-K: Immunostain.

B: CD23 $(4 x)$.

D: CD20 (4x).

E, F: BCL6 (E: 4x; F: 40x).

G: CD10 (10x).

H, I: CD3 (H: 10x; I: 40x)

J, K: BCL2 (J: 10x; K: 40x).

\section{Discussion}

FL is thought to arise from the follicular center B lymphocytes; therefore, the neoplastic cells express pan-B antigens and germinal center B cell markers CD10 and BCL6. The characteristic translocation $\mathrm{t}(14 ; 18)$ leads to upregulation of BCL2 protein conferring anti-apoptotic advantage to germinal center B cells which form neoplastic follicles via interacting with $\mathrm{T}$ cells and follicular dendritic cells, although it appears that BCL2 rearrangement by itself is not enough to result in lymphoma formation and additional genetic abnormalities are required [5]. The flow cytometry analysis of this patient's fine needle aspiration from the left inguinal lymph node revealed an abnormal light chain negative cell population with a differential diagnosis including FL, diffuse large B-cell lymphoma, and Burkitt lymphoma. Nevertheless, the effacement of 
normal lymph node architecture by closely packed and relatively uniform follicles that lack a mantle zone, the positive immunostain of the follicle cells for BCL6, CD10, and BCL2, and negative MYC translocation support the diagnosis of FL. The IgH/BCL2 translocation was not detected in this case by FISH even though BCL2 expression was identified by immunohistochemistry. The translocation $\mathrm{t}(14 ; 18)(\mathrm{q} 32 ; \mathrm{q} 21)$ is a genetic hallmark of FL, but its presence in FL depends on the technique used. Even with the most sensitive FISH assay, it has been reported that BCL2 translocation can be detected in $64 \%$ to $100 \%$ of FL $[6,7]$.

The overexpression of BCL2 protein can be present or absent in FL without $\mathrm{t}(14 ; 18)$. In a study of $49 \mathrm{FL}$ cases without $\mathrm{t}(14 ; 18)$, BCL2 protein overexpression was present in 33\% of the cases [8]. In another study involving $164 \mathrm{FL}$ cases, 17 cases were identified to be without $t(14 ; 18)$, among which 6 cases were positive for BCL2 at the protein level [9]. Interestingly, FL with and without $t(14 ; 18)$ showed distinct genetic features, gene expression and immunohistochemical profiles, but the FL without t $(14 ; 18)$ still belonged to the biological spectrum of FL. The absence of surface light chain expression in our FL case warrants its differentiation from precursor B- cell acute lymphoblastic leukemia (B-ALL), which is ruled out by negative TdT and CD34 expression. The morphology on H\&E also strongly argues against B-ALL. sIg light chain restriction in B cells is especially helpful in documenting clonality and loss of sIg light chain is a rare event in B-cell lymphoma. The frequency of sIg light chain loss ranges from $3.4 \%$ to $12.2 \%$, depending on the criteria used in the studies $[3,4]$. The absence of sIg on malignant B cells can result from any defect at the levels from gene transcription to transportation of fully assembled protein to the cell surface. In a study of 36 lymphoma cases, it was found that complete absence of sIg light chain in a mature B cell proliferation can be used as a surrogate marker to help diagnose peripheral B cell lymphoma. However, this concept is cautioned by the finding of benign follicular hyperplasia with expansion of sIg light chain-negative B cells without clonality [10].

Our case of FL not only showed loss of surface and cytoplasmic immunoglobulin light chain expression, but it also displayed negative clonal IGH rearrangement by PCR. This could result from the extensive and ongoing somatic hypermutation of the variable region genes on the immunoglobulin heavy and light chains, hampering PCR primer annealing. It was found in a study that only $86 \%$ of 109 definitive FL cases demonstrated clonality at the IGH locus, while $100 \%$ of these cases showed clonality when IGH locus PCR assessment was combined with IGK and/or IGL PCR [11]. Furthermore, immunoglobulin heavy chain rearrangement was not detected among $29 \%$ of the sIg negative B cell lymphoma cases [3]
Also, this case demonstrated a cytogenetic abnormality of $46, X X$, $-12,+$ mar [6]. This case represents the first known reported case with this constellation of findings.

\section{Conclusion}

The case presented here is that of a low-grade FL with the following highly unusual ancillary testing results: lack of surface or cytoplasmic immunoglobulin light chain expression, negative immunoglobulin heavy chain rearrangement by PCR, and negative $t(14 ; 18)$ by FISH. While the H\&E is typical for a low-grade FL with a follicular growth pattern, it is highly unusual for all the studies to be negative, coupled with the abnormal cytogenetic finding of 46 , $\mathrm{XX},-12,+\operatorname{mar}[6]$

\section{Conflict of Interest}

The authors declare that there is no conflict of interest

\section{References}

1. Anderson JR, Armitage JO, Weisenburger DD (1998) Epidemiology of the non-Hodgkin's lymphomas: distributions of the major subtypes differ by geographic locations. Ann Oncol 9(7): 717-720.

2. Groves FD, Linet MS, Travis LB, Devesa SS (2000) Cancer surveillance series: non-Hodgkin's lymphoma incidence by histologic subtype. J Natl Cancer Inst 92(15): 1240-1251.

3. Li S, Eshleman JR, Borowitz MJ (2002) Lack of surface immunoglobulin light chain expression by flow cytometric immunophenotyping can help diagnose peripheral B-cell lymphoma. Am J Clin Pathol 118(2): 229-234.

4. Kaleem Z, Zehnbauer BA, White G, Zutter MM (2000) Lack of expression of surface immunoglobulin light chains in B-cell non-Hodgkin lymphomas. Am J Clin Pathol 113(3): 399-405.

5. Kridel R, Sehn LH, Gascoyne RD (2012) Pathogenesis of follicular lymphoma. J Clin Invest 122(10): 3424-3431.

6. Gu K, Chan WC, Hawley RC (2008) Practical detection of t (14;18) (IgH/ BCL2) in follicular lymphoma. Arch Pathol Lab Med 132(8): 1355-1361.

7. Vaandrager JW, Schuuring E, Raap T, Philippo K, Kleiverda K, et al. (2000) Interphase FISH detection of BCL2 rearrangement in follicular lymphoma using breakpoint-flanking probes. Genes Chromosomes Cancer 27(1): 85-94.

8. Horsman DE, Okamoto I, Ludkovski O, Le N, Harder L, et al. (2003) Follicular lymphoma lacking the $t(14 ; 18)$ (q32; q21): identification of two disease subtypes. Br J Haematol 120(3): 424-433.

9. Leich E, Salaverria I, Bea S, Zettl A, Wright G (2009) Follicular lymphomas with and without translocation $t(14 ; 18)$ differ in gene expression profiles and genetic alterations. Blood 114(4): 826-834.

10. Zhao XF, Cherian S, Sargent R, Seethala R, Bonner H, et al. (2005) Expanded populations of surface membrane immunoglobulin light chain-negative B cells in lymph nodes are not always indicative of B-cell lymphoma. Am J Clin Pathol 124(1): 143-150.

11. Evans PA, Pott Ch, Groenen PJ, Salles G, Davi F, et al. (2007) Significantly improved PCR-based clonality testing in B-cell malignancies by use of multiple immunoglobulin gene targets. Leukemia 21(2): 207-214. 


\section{ISSN: 2574-1241}

DOI: $10.26717 /$ BJSTR.2020.26.004309

Gabriele De Sena. Biomed J Sci \& Tech Res

(C) This work is licensed under Creative

Submission Link: https://biomedres.us/submit-manuscript.php

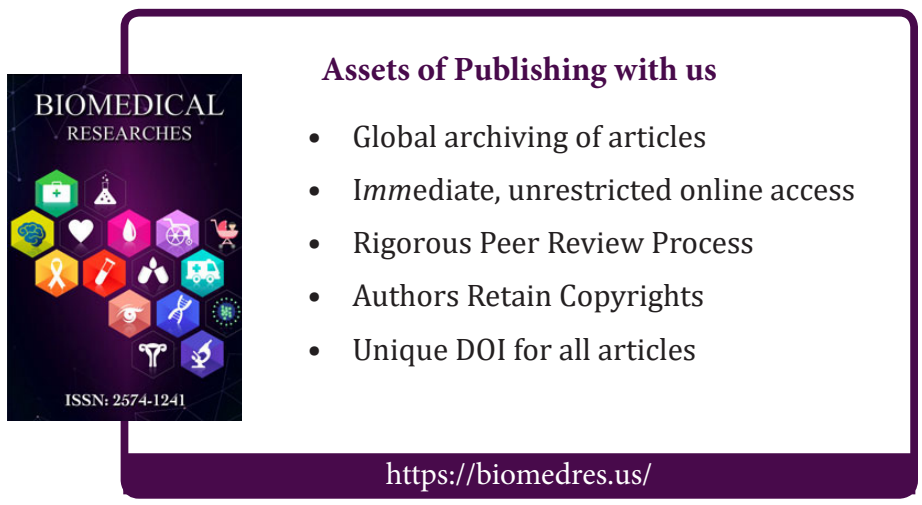

